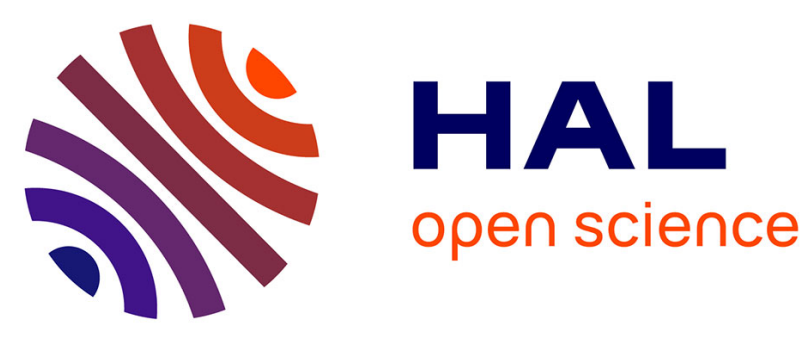

\title{
Scheduling, Binding and Routing System for a Run-Time Reconfigurable Operator Based Multimedia Architecture
}

Erwan Raffin, Christophe Wolinski, François Charot, Krzysztof Kuchcinski, Stéphane Guyetant, Stéphane Chevobbe, Emmanuel Casseau

\section{To cite this version:}

Erwan Raffin, Christophe Wolinski, François Charot, Krzysztof Kuchcinski, Stéphane Guyetant, et al.. Scheduling, Binding and Routing System for a Run-Time Reconfigurable Operator Based Multimedia Architecture. Design and Architectures for Signal and Image (DASIP), Oct 2010, Edinburgh, United Kingdom. inria-00539874

\section{HAL Id: inria-00539874 \\ https://hal.inria.fr/inria-00539874}

Submitted on 25 Nov 2010

HAL is a multi-disciplinary open access archive for the deposit and dissemination of scientific research documents, whether they are published or not. The documents may come from teaching and research institutions in France or abroad, or from public or private research centers.
L'archive ouverte pluridisciplinaire HAL, est destinée au dépôt et à la diffusion de documents scientifiques de niveau recherche, publiés ou non, émanant des établissements d'enseignement et de recherche français ou étrangers, des laboratoires publics ou privés. 


\title{
Scheduling, Binding and Routing System for a Run-Time Reconfigurable Operator Based Multimedia Architecture
}

\author{
E. Raffin* ${ }^{*}$, Ch. Wolinski* ${ }^{*}$, F. Charot ${ }^{\dagger}$, K. Kuchcinski ${ }^{\S}$, S. Guyetant ${ }^{\ddagger}$, S. Chevobbe ${ }^{\ddagger}$ and E. Casseau*† \\ *University of Rennes I. Rennes, France. Email: wolinski@irisa.fr \\ ${ }^{\dagger}$ INRIA. Rennes, France. Email: charot@irisa.fr \\ $\ddagger$ CEA, LIST. Gif-sur-Yvette, France. Email: stephane.guyetant@cea.fr \\ $\S$ Dept. of Computer Science, Lund University, Sweden. Email: krzysztof.kuchcinski@cs.lth.se \\ TTechnicolor Research \& Innovation, Rennes, France. Email: erwan.raffin@technicolor.com
}

\begin{abstract}
This paper presents a system for application scheduling, binding and routing for a run-time reconfigurable operator based multimedia architecture (ROMA). We use constraint programming to formalize our architecture model together with a specific application program. For this purpose we use an abstract representation of our architecture, which models memories, reconfigurable operator cells and communication networks. We also model network topology. The use of constraints programming makes it possible to model the application scheduling, binding and routing as well as architectural and temporal constraints in a single model and solve it simultaneously. We have used several multimedia applications from the Mediabench set to evaluate our system. In $78 \%$ of cases, our system provides results that are proved optimal.
\end{abstract}

Keywords-Constraint Programming; Reconfigurable Architectures; Embedded Platforms for Multimedia;

\section{INTRODUCTION}

Nowadays, modern highly parallel architectures are composed of many parallel processing units (homogenous or heterogenous) connected through communication networks. The processing elements are usually equipped with local memories. For about ten years, they have been containing, more and more frequently, run-time reconfigurable data-paths well tailored for selected classes of specific applications. These architectures are very efficient but tools to compile application programs for these new application-specific architectures are still missing. To do application scheduling, binding and routing for such systems is not a trivial task. To tackle this problem, we proposed a new generic system based on the constraint programing paradigm (CP) that enables execution of all above discussed tasks simultaneously. For the purpose of this paper, our system was adopted to support a run-time reconfigurable operator based multimedia architecture (ROMA).

The general flow of the proposed system is presented in Figure 1. It uses a generic compilation platform GECOS recently extended with polyhedral transformations [1]. Our system uses Hierarchical Conditional Dependency Graphs $(H C D G s)$ as the internal design representation. $H C D G$ captures both the application control-flow and data-flow [2], [3]. It supports formal graph transformations and optimizations.

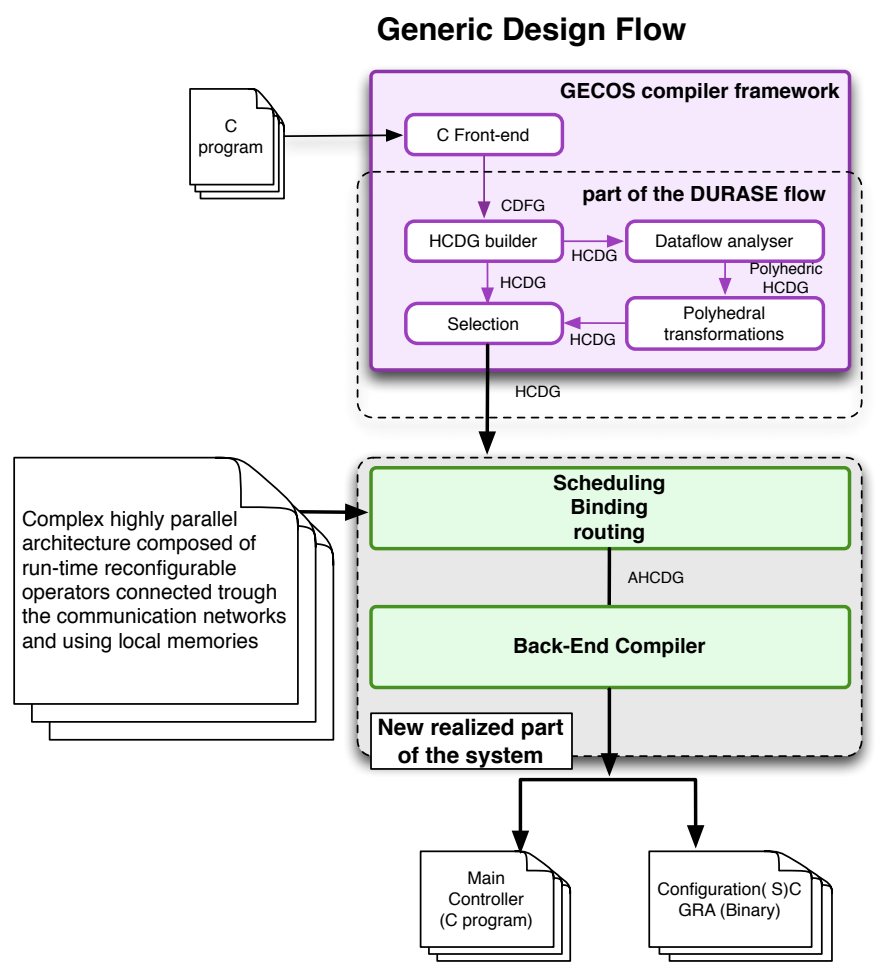

Fig. 1. Global design flow overview.

In the $H C D G$, graph nodes are guarded by boolean conditions and polyhedrons depending on loop indexes and parameters. After data-flow analysis [4], each read statement in a graph has a set of possible source contributions relying on its execution contexts and polyhedral conditions. Finally, loops are unrolled totally or partially to generate an $H C D G$ which is an input to the remainder part of the system.

As shown in Figure 1, the inputs to our system are an application program written in $\mathrm{C}$ and an abstract generic parallel run-time reconfigurable architecture model. The outputs are the $\mathrm{C}$ program and the configuration information (binary files) needed to manage the run-time reconfigurable ROMA 
architecture.

The newly developed system, presented in this paper, is part of the DURASE system [5], [6] (see Figure 1). It implements our new method, based on CP, that enables to model complex run-time reconfigurable architectures together with their application programs. The model can then be used to perform scheduling, binding and routing while optimizing application's execution time. Our system contains also the target dependent back-end compiler (in our case, the supporting ROMA architecture).

This paper is organized as follows. In section II, related works on scheduling and mapping are discussed. The targeted architecture and its abstract model is presented in section III and III-E. Section IV introduces briefly constraint programming, that is used in our approach. The constraint programming model to solve the resource constraint scheduling, binding and routing simultaneously for the abstract architecture model is discussed in V. Section VI presents experimental results. Finally, conclusions are presented in section VII.

\section{RELATED WORK}

Application scheduling, binding and routing for a run-time reconfigurable architectures are complex problems. Each of these problems is known to be NP-complete. In general two trends are dominant to deal with these kind of problems, both coming from high-level synthesis (HLS) domain.

First trend represents simple and dedicated algorithms to solve practical instances of the problem effectively and efficiently. List scheduling, for example, and its derivatives are the most commonly used and adapted algorithms. In [7], near optimal results are obtained quickly using a list-based scheduler thanks to a tight cohesion between partitioning and scheduling steps. More recently, [8] targets an industrial distributed reconfigurable instruction cell based architecture considering binding and routing effect, register assignment and operation chaining. Branch-and-Bound algorithm have been proposed in [9] and delivered good results but addressing scheduling and allocation separately.

Second trend represents methods for solving these problems optimally. Integer linear programming (ILP) and Mixed integer programming (MIP) are common methods for mixed constrained version of these problems. They are also frequently used to compare results from heuristic-based methods. The contribution presented in [10] is particularly in line with our work because an ILP formulation for solving optimally and simultaneously the mapping, routing and scheduling of a given data flow graph on a coarse-grain architecture is presented. The main difference, apart the formulation type, resides in the targeted architecture model, which consists of homogeneous processing elements and mesh-like interconnection network. In [11], a MIP formulation is proposed for optimal mapping of digital signal processing algorithms on commercially VLIW DSPs, both homogeneous and heterogeneous architecture are addressed.

The constraint programming (CP) approach is one of the most relevant in the second trend and particularly developed during last years for different purpose. Kuchcinski and Wolinski, have widely participated in this development through their research, mainly in HLS [3], [12], [13]. More recently they also contributed to generation of optimized application specific reconfigurable processor extensions [14], [15]. This approach has also been used in the context of embedded distributed real-time systems [16].

According to a recent survey on methods and tools for embedded reconfigurable systems [17], CP and evolutionary/genetic algorithms presented in [18] belong to the most promising approaches to efficiently produce high-quality solutions for the complex architecture synthesis problems involving multi-objective optimization. To illustrate this, [19] compares three static schedulers. First one, list-based, has high efficiency but the least accuracy; the second, based on $\mathrm{CP}$, ensures optimal solutions but with significant searching effort; the last one, based on a genetic algorithm, shows reasonable efficiency and better accuracy than the first one.

To our knowledge, this is the first paper addressing the problem of solving simultaneously binding, routing and scheduling a data flow graph for the run-time reconfigurable operator based embedded architecture using CP. With this approach, it is possible to solve simultaneously application binding, routing and scheduling problems under architectural and temporal constraints. Moreover, we use the JaCoP solver [20], which provides classical primitive constraints but also conditional and highly efficient global ones particularly useful for our problem formulation.

\section{DESCRIPTION OF ROMA PROCESSOR}

The ROMA processor is composed of a set of coarse grain reconfigurable operators, data memories, configuration memories, operator network, data network, control network and a centralized controller. The centralized controller manages the configuration steps and the execution steps. The ROMA processor has three different interfaces: one data interface connected to the operator network, one control interface and one debug interface connected to the main controller.

\section{A. Reconfigurable datapath}

The reconfigurable datapath of the ROMA processor is made up of a set of heterogeneous or homogeneous reconfigurable operators. Their number can be statically changed from 4 to 12 depending on the computing power needed for a particular application domain. Each reconfigurable operator has its own configuration memory and its own control interface to the main controller, each of them being able to be configured and controlled independently.

These reconfigurable operators are connected together via a dedicated network (called operator-operator network) and to the data memories via another network (called data memoryoperator network). The local memories have their own programmable address generators. Figure 2 shows the block diagram of the ROMA processor. The main controller (Global CTRL) executes a $\mathrm{C}$ program defining synchronisations between the configuration and execution sequences. 


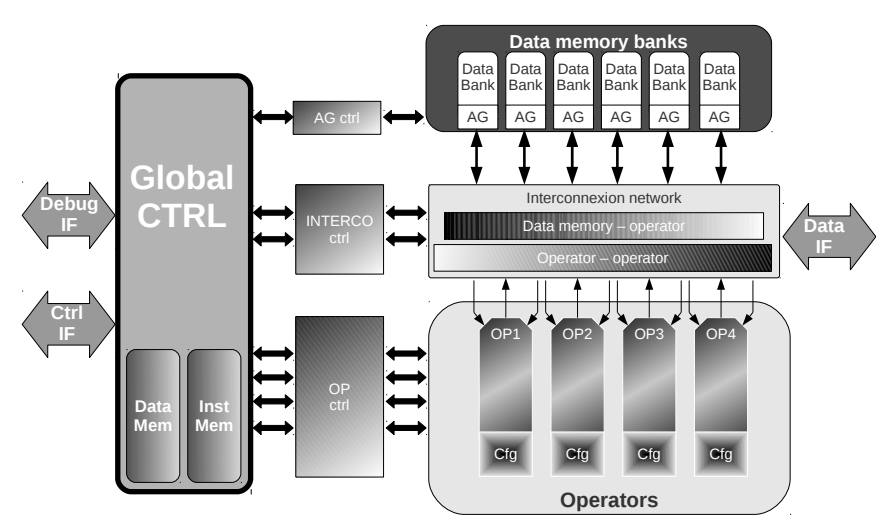

Fig. 2. Architecture of ROMA processor : the control structure includes a Global CTRL and dedicated controllers designated for each module of the reconfigurable datapath. The reconfigurable datapath is composed of data memory banks, two interconnection networks and a set of coarse grain reconfigurable operators.

\section{B. Coarse grain reconfigurable operator}

The coarse grain reconfigurable operators are four pipeline stage operators that can execute standard arithmetic (such as ADD, SUB, MUL, ABS), logic (such as AND, OR, XOR) and shift operations. The operators can also compute accumulation operations (such as SAD and MAC). The datapath of the operators has $32 / 40$ bit wide inputs and outputs. The multiplier is a $16 \times 16$ bit multiplier that can be split into two $8 \times 8$ bit multipliers.

\section{Interconnection networks}

The transfers inside the ROMA processor are organized according to the type of communication. There are data, configuration and control communications and the interconnection networks were split according to these types. These interconnection networks allow partial reconfiguration, to prepare the next communication pattern without stopping the execution. The interconnection networks are configurable in one cycle.

\section{Data interconnection network}

The data transfers can be done either between data memories and operators or between operators themselves. Dedicated interconnection networks were designed to connect data memories to operators (data memory - operator network) and operators together (operator - operator network). The data width is parametrizable between 32 bit and 40 bit according to the needs. The data interconnection does not add any clock cycles during the communication, but a fixed latency between 1 and 3 cycles according to the number of operators and data memory banks. A communication pattern is defined by two configurations, for both types of networks. The goal of this solution is to minimize the hardware complexity of the interconnection network and avoid a costly crossbar interconnection.

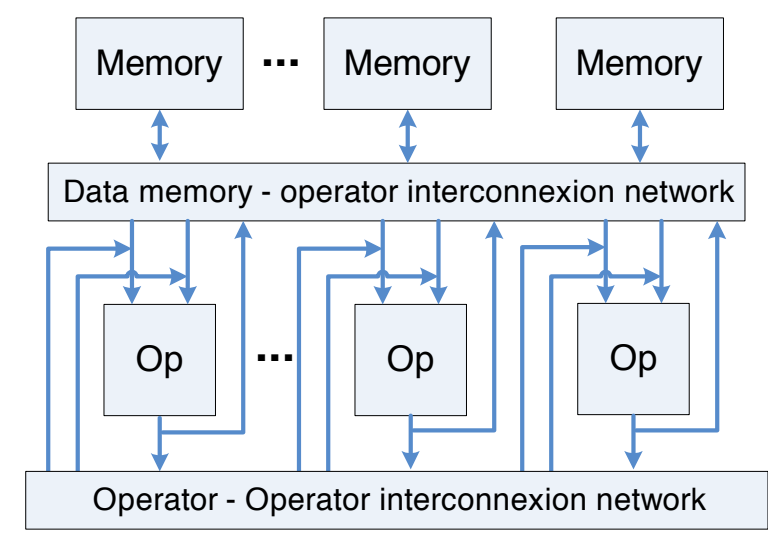

Fig. 3. Generic architecture model.

1) Operator - operator interconnection network: The operator - operator interconnection network is able to achieve all acyclic connection patterns between reconfigurable operators, connecting $N$ operators with 2 inputs and 1 output. This interconnection network is optimized to minimize the hardware cost and the latency. Each output of each operator can be connected to each $N / 2$ inputs of the next operators (i.e., operators with higher identification number). An output can be connected to several inputs of the next operators. Each connector is configured by one bit.

2) Data memory - operator interconnection network: The data memory - operator interconnection network has to support connections between data memory banks and the operators and can achieve several communication patterns simultaneously. This flexibility is needed to ease the data placement in the data memory banks. This interconnection network should minimize the critical path. Thus, 1 to 3 latency cycles are needed to cross the data memory - operator interconnection network. These latency cycles are managed during the compilation of an application. The latency is variable according to the distance between the memory bank and the operators that are connected.

\section{E. Architecture Abstract Model}

The abstract architecture model of the ROMA architecture is depicted in Figure 3. It is composed of local memories, a memory-operator interconnection network, reconfigurable operators and a specific operator-operator interconnection network. The architecture is defined as follows.

- the number and size of the local memories are parametrized,

- each memory has one true port; it means that only one $\mathrm{read} / \mathrm{write}$ operation can be executed at the same time,

- the read/write operation latencies are constant,

- each memory is identified by a unique number,

- each operator is identified by a unique number,

- the operators can be heterogenous (each operator can execute a specific set of complex operations),

- each operator has at most two input ports and one output port, 
- the memory-operator interconnection network is a full crossbar network,

- the operator-operator interconnection network is parametrized; it can be defined by a connection matrix containing the information about point to point operator connections,

- the operator-operator interconnection network is characterized by a constant read/write latency.

This abstract architecture has been implemented in our framework as a meta-model with its own editor allowing easy and quick specification of the resource constraints. In this way, it is possible to specify the amount of functional resources and supported operations, each with a delay, amount of memory, etc.

\section{CONSTRAint PROgRAMming}

In our system, we use constraint satisfaction methods implemented in constraint programming environment JaCoP [20], [21]. Below we provide a very short introduction to constraint programming but the more thorough discussion can be found in [22], for example.

A constraint satisfaction problem (CSP) is defined as a 3-tuple $\mathcal{S}=(\mathcal{V}, \mathcal{D}, \mathcal{C})$ where $\mathcal{V}=\left\{x_{1}, x_{2}, \ldots, x_{n}\right\}$ is a set of variables, $\mathcal{D}=\left\{\mathcal{D}_{1}, \mathcal{D}_{2}, \ldots, \mathcal{D}_{n}\right\}$ is a set of finite domains (FD), and $\mathcal{C}$ is a set of constraints. Finite domain variables (FDV) are defined by their domains, i.e. the values that are possible for them. A finite domain is usually expressed using integers, for example $x:: 1 . .7$. A constraint $c\left(x_{1}, x_{2}, \ldots, x_{n}\right) \in \mathcal{C}$ among variables of $\mathcal{V}$ is a subset of $D_{1} \times D_{2} \times \ldots \times D_{n}$ that restricts which combinations of values the variables can simultaneously take. Equations, inequalities and even programs can define a constraint.

A solution to a CSP is an assignment of a value from variable's domain to every variable, in such a way that all constraints are satisfied. The specific problem to be modeled will determine whether we need just one solution, all solutions or an optimal solution given some cost function defined in terms of the variables.

The solver is built using constraints own consistency methods and systematic search procedures. Consistency methods try to remove inconsistent values from the domains in order to reach a set of pruned domains such that their combinations are valid solutions. Each time a value is removed from a FD, all the constraints that contain that variable are revised. Most consistency techniques are not complete and the solver needs to explore the remaining domains for a solution using search. Solutions to a CSP are usually found by systematically assigning values from variables domains to the variables. It is implemented as depth-first-search. The consistency method is called as soon as the domains of the variables for a given constraint are pruned. If a partial solution violates any of the constraints, backtracking will take place, reducing the size of the remaining search space.

The constraint programming approach has been recently used in UPaK (Abstract Unified Patterns Based Synthesis Kernel for

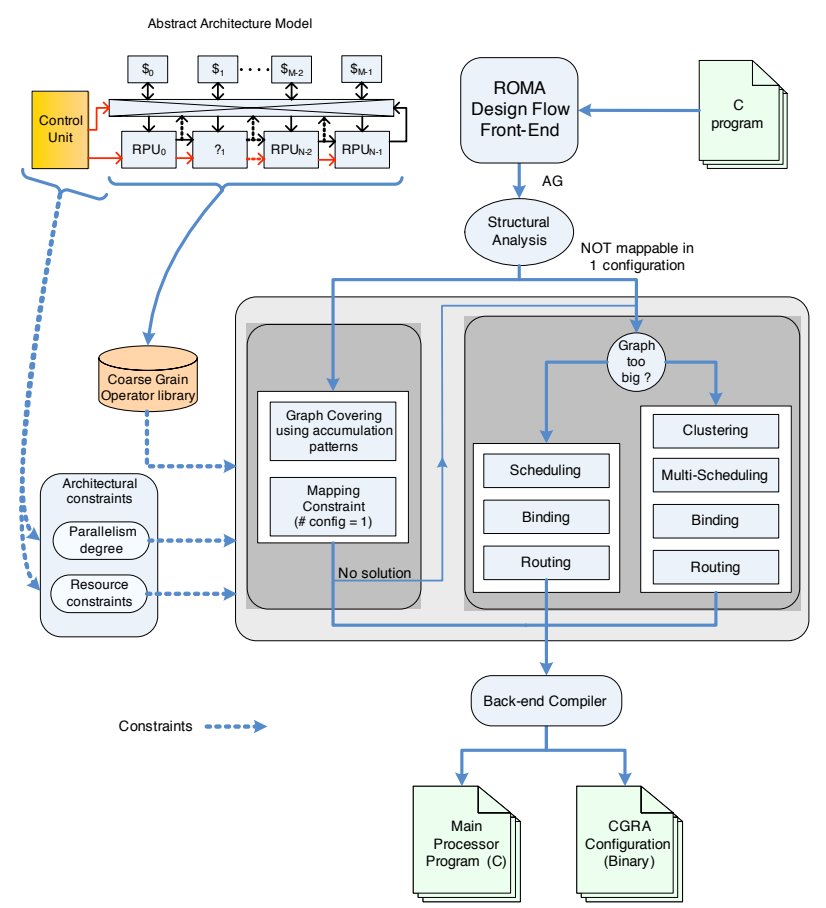

Fig. 4. Detailed scheduling, binding and routing and flow.

Hardware and Software Systems) [15] for automatic design of application-specific reconfigurable processor extensions.

\section{Scheduling AND Binding Constraint Model}

The place of scheduling, binding and routing steps in the whole design flow are presented in detail in Figure 4. The inputs for these steps are the application graph (AG), the library of the functionally reconfigurable operators and the architectural constraints (derived from the ROMA multi-media architecture). The outputs are the main processor program (C file) and the configuration information (binary files).

The ROMA architecture supports two modes of execution. The first one corresponds to the data flow model of execution. This mode can be used when only one configuration is used during the processing. In the second mode, the configuration of the communication networks and configuration of operators can be changed each cycle. In this paper, we consider both modes of execution but the accumulation operations available in mode one are not considered.

\section{A. Application Graph}

Formally, the AG is modeled as a direct acyclic graph $A G=$ $(N, E)$ where each node $n \in N$ represents a computation $\left(n \in O P_{s}\right)$ or an input/output memory access $(n \in I O s)$ and each direct edge $e \in E$ a data transfer.

\section{B. Finite Domain Variables Definition}

In order to model the scheduling, binding and routing we use the following primary FDVs defined for all nodes $n \in N$ and all edges $e \in E$.

- $n_{\text {start }}::\{0 . . \infty\}$ defines the start time of processing of node $n$, 
- $n_{\text {delay }}$ defines the processing time of node $n$ (in the ROMA processor an execution time of node $n$ is constant for all reconfigurable operators),

- $n_{\text {end }}::\{0 . . \infty\}$ defines the processing completion time of node $n$,

- $n_{o p}::\left\{i \mid o p_{i} \in\{0 . . \mid\right.$ operator $s \mid-1\} \wedge$ op $_{i}$ can execute $n\}$ defines the operator binding to node $n$,

- $n_{o p_{\text {activity }}}::\{0 . . \infty\}$ defines the time when operator op is occupied to execute node $n \in O P_{s}$; this time includes node processing time and the time needed to transfer the data,

- $n_{\text {mem }}::\{0 . . \mid$ memories $\mid-1\}$ defines the memory used to store the data from node $n$,

- $n_{\text {start }_{W R}}::\{0 . . \infty\}$ defines the starting time of the memory data write operation for the data coming from node $n$,

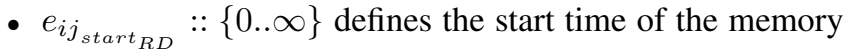
data read operation for the computation represented by node $n_{j}$, (data produced by node $n_{i}$ ),

- $n_{\text {store }_{M_{n}}}::\{0,1\}$ defines whether the data from node $n$ needs to be saved in memory $M_{n}$ (value 1) or not (value $0)$,

- $n_{\text {life_time }}::\{0 . . \infty\}$ defines the life time of the data produced by node $n$,

- $e_{\text {mem_ope }}::\{0,1\}$ defines whether the memory-operator interconnection network is used (value 1 ) for the data transfer represented by edge $e$,

- $e_{\text {ope_ope }}::\{0,1\}$ defines whether the operator-operator interconnection network is used (value 1) for the data transfer represented by edge $e$.

Secondary FDVs variables are introduced, when needed, later in this paper..

\section{Communication Constraints}

In $A G$, an edge represents a data transfer on one of the two communication networks, either through the memoryoperator interconnection network or through the operatoroperator interconnection network. For each edge $e \in E$ we define exclusive choice of the network using constraint (1).

$$
\forall e \in E: e_{\text {mem_ope }}+e_{\text {ope_ope }}=1
$$

If an edge represents a data transfer from an input node to a computation node, then we impose constraint (2) to use the memory-operator interconnection network. Similar constraints are imposed for data transfers from a computation node to an output node.

$$
\begin{aligned}
& \forall e_{i j}=\left(n_{i}, n_{j}\right) \in E \mid n_{i} \in I O s \wedge n_{j} \in O P s \vee \\
& n_{i} \in O P s \wedge n_{j} \in I O s: \\
& e_{i, j_{\text {mem_ope }}}=1
\end{aligned}
$$

In the ROMA architecture, the memory-operator interconnection network is implemented as a full crossbar network. For this reason we do not need to impose any additional resource sharing constraints to model these connections.
On the contrary, the operator-operator interconnection network imposes some communication limitations according to the network topology presented in III-D1 and III-E. In this case, constraint (3) must be fulfilled for each edge $e_{i j}=$ $\left(n_{i}, n_{j}\right) \in E$ when $n_{i}, n_{j} \in O P s$.

$$
\text { If } e_{i j_{o p e \_o p e}}=1 \text { then } n_{j_{o p}}=n_{i_{o p}}+e_{i j_{o p_{r}}}
$$

Note that we introduced variable $e_{i j_{o p_{r}}}::\left\{1 . . \frac{\mid \text { operators } \mid}{2}\right\}$ for all edges. This variable models which operators (represented as operators' numbers) are reachable from operator $n_{i_{o p}}$. The finite domain of this variable can be equal to $\left\{0 . . \frac{\mid \text { operators } \mid}{2}\right\}$ if a loop back link on operator exists.

In general, to model the topology of a specific network, a dedicated communication matrix ComMat can be used. This matrix represents a relation between nodes $n_{i_{o p}}$ and $n_{j_{o p}}$ and contains information about all possible point to point connections between these nodes. In this case, constraint (3) is replaced by constraint (4). In practice, it is implemented as ExtensionalSupport constraint [21].

$$
\text { If } e_{i j_{o p e_{-} o p e}}=1 \text { then } n_{j_{o p}}=\operatorname{ComMat}\left[n_{i_{o p}}\right]
$$

\section{Timing Constraints}

The completion time of the processing of node $n \in O P s$ ( $n_{\text {end }}$ ) is defined by equation (5). Note that if $n \in I O s$ then $n_{\text {delay }}=0$.

$$
n_{\text {end }}=n_{\text {start }}+n_{\text {delay }}
$$

The node precedence relations imposed by the partial order of AG are modeled by constraints (6) or (7) depending on which communication network was selected for data transfer.

In order to transfer the data between two nodes $n_{i}, n_{j} \in$ OPs $\left(e_{i, j}=\left(n_{i}, n_{j}\right) \in E\right)$ through the memory-operator communication network, memory write and read operations must be modeled (see Figure 5). In the ROMA architecture, each operator produces one result that can be communicated to other nodes. Therefore, only one memory write operation for all output edges is modeled. The memory read operations are modeled for each out-coming edge $e \in n_{\text {outputs }}$ using the memory-operator communication network for data transfers.

The start times of memory write and read operations are modeled by FDVs $n_{i_{\text {start }_{W R}}}$ for all nodes $n_{i} \in N$ and $e_{i, j_{\text {start }} R D}$ for all edges from $n_{i}$, respectively. Inequality (6a) constraints the start time of a memory write operation in relation to the completion time of the operation represented by node $n_{i}$. In the same way, the memory read operation of the previously saved data is defined in constraint (6b). In this case, the read operation takes place when a corresponding write operations is completed. Finally, the next operation can begin only when its input data are loaded (constraint (6d)). The delays of both memory write and read operations, $\Delta e_{i j_{W R}}$ and $\Delta e_{i j_{R D}}$, are computed using constraints (6c) and (6e) respectively. This is done according to the write and 


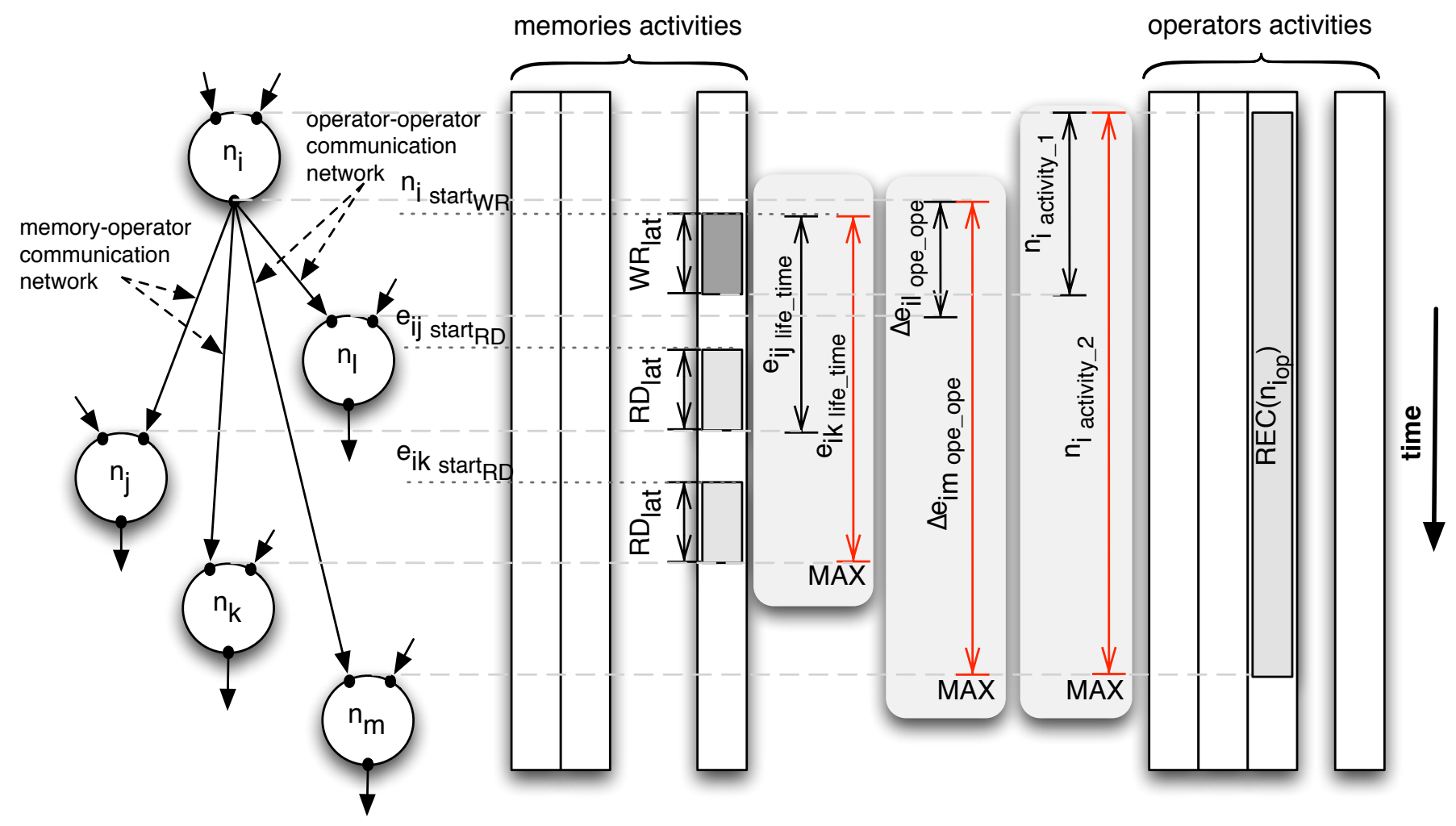

Fig. 5. Example with communications on both networks.

read latencies (denoted $W R_{\text {lat }}$ and $R D_{\text {lat }}$ ) derived from the architecture model and the constants considered here.

$$
\begin{aligned}
& n_{i_{\text {start } W R}} \geq n_{i_{\text {end }}} \\
& e_{i j_{\text {start }_{R D}}} \geq n_{i_{\text {start } W R}}+\Delta e_{i j_{W R}} \\
& \Delta e_{i j_{W R}}=W R_{\text {lat }} * e_{i, j_{\text {mem_ope }}} \\
& e_{i j_{\text {start }_{R D}}}+\Delta e_{i j_{R D}}=n_{j_{\text {start }}} \\
& \Delta e_{i j_{R D}}=R D_{\text {lat }} * e_{i, j_{\text {mem_ope }}}
\end{aligned}
$$

The precedence constraints between nodes $n_{i}$ and $n_{j} \in$ OPs $\left(e_{i j}=\left(n_{i}, n_{j}\right) \in E\right)$ imposed when a data transfer is done through the operator-operator network are specified by formulas (7), where FDV $\Delta e_{i j_{\text {ope ope }}}::\{0 . . \infty\}$ represents the time when the network is occupied. We consider that the operator-operator interconnection network is occupied from the completion time of the processing of source node $n_{i}$ to the start time of the processing of destination node $n_{j}$. This is formalized by (7a). In (7b) the operator-operator interconnection network latency ope_ope $e_{\text {lat }}$, derived from the architecture model is used to constrain the occupation time. Variable $n_{i_{\text {net_acces }}}=1$ if at least one data transfer through the operator-operator interconnection network is executed.

$$
\begin{aligned}
& \Delta e_{i j_{\text {ope_ope }}}=n_{j_{\text {start }}}-n_{i_{\text {end }}} \\
& \Delta e_{i j_{\text {ope_ope }}} \geq \text { ope_ope } e_{\text {lat }} * e_{i j_{\text {ope_ope }}} \\
& n_{i_{\text {net_acces }}} \in\{0,1\} \Leftrightarrow \sum_{\forall e_{i j} \in n_{i_{\text {outputs }}}} e_{i j_{\text {ope } o p e}}>0
\end{aligned}
$$

\section{E. Resource Sharing Constraints}

In our CP model, we model the resources sharing process by using the combinatorial constraint Diff2 presented in [20]. This constraint prohibits simultaneous usage of resources by imposing relations between a set of $2 \mathrm{D}$ rectangles in a timeresource space. These 2D rectangles are defined with a syntax $R e c=[x, y, \Delta x, \Delta y]$. Variables $y, x$ and $\Delta x$ represent the resource number, the operation start time and the occupation time of the resource, respectively. In general, $\Delta y=1$ if a resource is used and $\Delta y=0$ otherwise. For each type of resource (as operator, memory etc.), we define a separate Diff2 constraint.

a) Memory Unit Activity Modeling: In order to model the potential memory access for each node $n_{i}$, we defined one rectangle corresponding to the memory write operation $\operatorname{Rec}\left(n_{i_{W R}}\right)$ (8a) and one rectangle corresponding to the memory read operation $\operatorname{Rec}\left(e_{i j_{R D}}\right)$. They are defined for each outcoming edge $e_{i j}$ (8c) from node $n_{i}$.

For rectangle $\operatorname{Rec}\left(n_{i_{W_{R}}}\right)$ (8b), $\Delta y$ is replaced by variable $n_{i_{\text {mem_access }}}$ that is equal 1 if at least one output edge represents a data transfer to the memory. For rectangle $\operatorname{Rec}\left(e_{i j_{R D}}\right)$, $\Delta y$ is replaced by variable $e_{i j_{\text {ope }} \text { ope }}$.

$$
\begin{aligned}
& \operatorname{Rec}\left(n_{i_{W R}}\right)=\left[n_{i_{\text {start } \mathrm{K}}}, n_{i_{\text {mem }}}, W R_{\text {lat }}, n_{i_{\text {mem_access }}}\right] \\
& n_{i_{\text {mem_acces }}} \in\{0,1\} \Leftrightarrow \sum_{\forall e_{i j} \in n_{i_{\text {outputs }}}} e_{i j_{\text {mem_ope }}}>0 \\
& \operatorname{Rec}\left(e_{i j_{R D}}\right)=\left[e_{i_{\text {start }_{R D}}}, n_{i_{\text {mem }}}, R D_{\text {lat }}, e_{i j_{\text {mem_ope }}}\right]
\end{aligned}
$$


TABLE I

RESULTS OBTAINED FOR RESOURCE-CONSTRAINED SCHEDULING AND MAPPING FOR SELECTED MULTIMEDIA APPLICATIONS.

\begin{tabular}{|c|c|c|c|c|c|c|c|c|c|}
\hline Application & $\overline{\mathrm{DFG}}$ & nodes & edges & input nodes & output nodes & Cycles & Optimal & Runtime (ms) & Time Out $(\mathrm{s})$ \\
\hline JPEG IDCT (col) & 1 & 35 & 40 & 13 & 4 & 16 & yes & 7693 & 30 \\
\hline$-/ /-$ & 2 & 57 & 65 & 22 & 5 & 26 & yes & 15117 & 30 \\
\hline Total DFGs for JPEG IDCT (col & $1+2$ & 92 & 105 & 35 & 9 & 42 & yes & 22810 & 30 \\
\hline JPEG IDCT (row) & 3 & 106 & 127 & 34 & 17 & 29 & no & TO & 30 \\
\hline Write BMP Header & 4 & 73 & 72 & 29 & 16 & 13 & yes & 875 & 10 \\
\hline$-/ /-$ & 5 & 19 & 18 & 8 & 4 & 5 & yes & 15 & 10 \\
\hline$-/ /-$ & 6 & 27 & 26 & 12 & 4 & 9 & yes & 47 & 10 \\
\hline$-/ /-$ & 7 & 27 & 26 & 12 & 4 & 9 & yes & 46 & 10 \\
\hline$-/ /-$ & 8 & 9 & 8 & 4 & 2 & 5 & yes & 0 & 10 \\
\hline Total DFGs for Write BMP Header & $4+. .+8$ & 155 & 150 & 65 & 30 & 41 & yes & 983 & 10 \\
\hline sobel 7x7 (unrolled 2x2) & 9 & 52 & 54 & 24 & 2 & 24 & yes & 360 & 10 \\
\hline MESA Matrix Mul & 10 & 52 & 60 & 20 & 4 & 16 & no & TO & 30 \\
\hline IIR biquad $\mathrm{N}$ sections (unrolled $\mathrm{x} 4$ ) & 11 & 66 & 73 & 29 & 1 & 55 & no & TO & 30 \\
\hline Roma H filter & 12 & 43 & 42 & 21 & 2 & 28 & yes & 297 & 10 \\
\hline
\end{tabular}

Constraint (9), defined for all nodes $n_{i} \in N$ and for all outcoming edges $e_{i j} \in n_{i_{\text {outputs }}}$, ensures exclusive access to the memory.

$$
\operatorname{Diff2}\left(\left[\ldots \operatorname{Rec}\left(n_{i_{W R}}\right), \operatorname{Rec}\left(e_{i j_{R D}}\right) \ldots\right]\right)
$$

To handle the possibility of performing several memory read operations on the same data at the same time, we defined Diff2 exceptions (10). These exceptions define possibility for some rectangles to overlap and are specified by a list of rectangle pairs $\left[R e c_{j}, \operatorname{Rec}_{k}\right]$.

$$
\begin{aligned}
& \forall n_{i} \in E \wedge e_{i j}, e_{i k} \in n_{i_{\text {outputs }}} \wedge j \neq k \\
& {\left[\operatorname{Rec}_{j}, \operatorname{Rec} c_{k}\right]=\left[\operatorname{Rec}\left(e_{i j_{R D}}\right), \operatorname{Rec}\left(e_{i k_{R D}}\right)\right]}
\end{aligned}
$$

For nodes representing input/output variables we do not define specific rectangles. Rectangles $\operatorname{Rec}\left(n_{W R}\right)$ and are not considered for input nodes and rectangles $\operatorname{Rec}\left(e_{i j_{R D}}\right)$ are not needed for output nodes.

b) Memory Unit Occupation Modeling: FDV variables $e_{i j_{\text {life_time }}}$ (defined for all edges) and $n_{i_{\text {life_time }}}$ (defined for all nodes in the AG) are used to model data life-time in memory. We consider that data produced by node $n_{i}$ transferred via memory $n_{i_{m e m}}$ occupies memory from the start time of its write operation until completion of the last read operation. The life-time of the data represented by $e_{i j}$ is expressed by the constraint (11a). According to the fact that all edges from a node represent data transfers of a unique data, we can simply define the life-time of the data produced by node $n_{i}$ by the constraint (11b).

$$
\begin{aligned}
& e_{i j_{\text {life_time }}}=n_{j_{\text {start }}}-n_{i_{\text {start } W R}} \\
& n_{i_{\text {life_time }}}=\max \left(\ldots, e_{i j_{l_{\text {ife_time }}}} * e_{i j_{\text {mem_ope }}}, \ldots\right) \\
& \text { where } \quad e_{i j} \in n_{i_{\text {outputs }}}
\end{aligned}
$$

In order to not exceed the memory size (m_size), we use the cumulative constraint Cumulative $\left(t, \Delta t, r a, m_{-}\right.$size $)$ [21], where variable $t$ defines, in our case, the start time of memory cell occupation, $\Delta t$ corresponds to the cell's occupation time and finally $\mathrm{ra}$ defines how many cells are used. $r a$ is 1 if memory $m_{i}$ is used to store the data (0 otherwise), in our case.

$$
\begin{aligned}
& \forall m_{i} \in\{0 . .|M e m|-1\}, \forall n_{i} \in N: \\
& \left(t_{i}=n_{i_{\text {start }_{W R}}} \wedge \Delta t_{i}=n_{i_{\text {life_time }_{\text {e }}}} \wedge\right. \\
& \left.m_{-} \text {used }_{i}::\{0,1\} \Leftrightarrow n_{i_{\text {mem }}}=m_{i}\right) \\
& \text { Cumulative }\left(t, \Delta t, m \_u s e d, m \_s i z e\right)
\end{aligned}
$$

c) Operator Unit Activity Modeling: Concerning the operator sharing constraints, we defined for each node $n_{i} \in O P s$ a rectangle modeling the operator activity. We do similar definitions for output operations. The time of operator activity $n_{i_{\text {op activity }}}$ is defined by constraint (14c). As we mentioned previously, the data can be transferred either by memoryoperator or operator-operator interconnection networks. Variables: $n_{i_{\text {activity_ } 1}}::\{0 . . \infty\}$ and $n_{i_{\text {activity_ }}}::\{0 . . \infty\}$ are used to model the operator unit occupation time for the first and the second networks, respectively. They are defined by constraints (14a) and (14b);

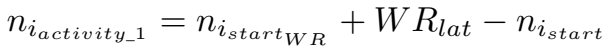

$$
\begin{aligned}
& n_{i_{\text {activity_2 }}}=\max \left(\ldots, \Delta e_{i j_{\text {ope_ope }}}, \ldots\right)+n_{i_{\text {delay }}} \\
& \text { where } e_{i j} \in n_{i_{\text {outputs }}} \\
& n_{i_{o p_{\text {activity }}}}=\max \left(n_{i_{\text {activity_ } 1}} * n_{i_{\text {mem_acces }}},\right. \\
& \left.n_{i_{\text {activity_2 }}} * n_{i_{\text {net_acces }}}\right)
\end{aligned}
$$

The rectangles representing operator activities and the corresponding Diff2 constraints are defined by (15a) and (15b).

$$
\begin{aligned}
& \forall n_{i} \in O P s: \operatorname{Rec}\left(n_{i_{o p}}\right)=\left[n_{i_{s t a r t}}, n_{i_{o p}}, n_{\left.i_{o_{p_{a c t i v i t y}}}, 1\right]}\right. \\
& \operatorname{Diff2}\left(\left[\operatorname{Rec}\left(n_{1_{o p}}\right), \operatorname{Rec}\left(n_{2_{o p}}\right), \ldots\right]\right)
\end{aligned}
$$

d) Cost Function: The cost function CostFunc that enables the optimization of the application time is defined by constraint (16).

$$
\text { CostFunc }=\max \left(\ldots, n_{i_{\text {end }}}, \ldots\right)
$$

This constraint makes it possibly to minimize the schedule lenght. It is defined for all AG output nodes. 


\section{EXPERIMENTAL RESULTS}

We have carried out extensive experiments to evaluate the quality of our method. All experiments have been run on $2 \mathrm{GHz}$ Intel Core Duo under the Windows XP operating system. In our experiments, the ROMA abstract model has been instantiated with 8 memories and 4 operators. All operators support the same types of computations and the delay of a computation is the same, independently to its resource assignment. The following latencies have been assumed $W R_{\text {lat }}=R D_{\text {lat }}=$ ope_ope $_{\text {lat }}=1$. We have also assumed that all data is stored in memories before processing starts.

Table I presents results obtained for applications from different multimedia benchmarks. Some of these applications are composed of several non-connected data flow graphs. Thus, the results are presented for all these non-connected subgraphs and for the whole application. The runtime includes the time necessary for finding the solution and the time needed to prove its optimality, if the optimality has been proved. Otherwise it is the time for finding the solution. In $78 \%$ of the cases, our system provides optimal results, confirming the high quality of our scheduling, binding and routing system.

\section{CONCLUSION}

Our new, $\mathrm{CP}$ based, scheduling, binding and routing system for run-time reconfigurable, coarse grain architectures has been proposed in this paper. The presented system was especially adopted for the ROMA architecture but the proposed abstract model is generic and supports more general interconnection networks than the ones derived from our specific reconfigurable architecture. This makes our model more general and applicable for other reconfigurable architectures. Thanks to the CP model of the abstract architecture, we could do the scheduling, binding and routing tasks simultaneously. This makes it possible to reach globally optimal solutions and obtain high quality results. For $78 \%$ of the considered multimedia applications, coming from the Mediabench set, our system generated optimal solutions and proved their optimality.

\section{ACKNOWLEDGMENT}

The work presented in this paper is supported by the French Architectures du Futur ANR program ANR-06-ARFU-004.

\section{REFERENCES}

[1] GeCoS, "Generic compiler suite - http://gecos.gforge.inria.fr/." [Online]. Available: http://gecos.gforge.inria.fr/

[2] A. A. Kountouris and C. Wolinski, "Efficient scheduling of conditional behaviors for high-level synthesis," ACM Trans. Des. Autom. Electron. Syst., vol. 7, no. 3, pp. 380-412, 2002.

[3] K. Kuchcinski and C. Wolinski, "Global approach to assignment and scheduling of complex behaviors based on hcdg and constraint programming," Journal of Systems Architecture, vol. 49, pp. 489 - 503, 2003.
[4] P. Feautrier, "Dataflow analysis of array and scalar references," International Journal of Parallel Programming, vol. 20, 1991.

[5] K. Martin, C. Wolinski, K. Kuchcinski, A. Floch, and F. Charot, "Constraint-driven identification of application specific instructions in the DURASE system," in SAMOS IX: International Workshop on Systems, Architectures, Modeling and Simulation, Samos, Greece, Jul. 2023, 2009.

[6] $\longrightarrow$ "Constraint-driven instructions selection and application scheduling in the DURASE system," in 20th IEEE International Conference on Application-specific Systems, Architectures and Processors (ASAP), Boston, USA, Jul.7-9, 2009.

[7] K. S. Chatha, "An iterative algorithm for hardware-software partitioning, hardware design space exploration and scheduling. design automation for embedded systems," Journal on Design Automation for Embedded Systems, vol. 5, pp. 281-293, 2000.

[8] Y. Yi, I. Nousias, M. Milward, S. Khawam, T. Arslan, and I. Lindsay, "System-level scheduling on instruction cell based reconfigurable systems," in DATE '06: Proceedings of the conference on Design, automation and test in Europe, 2006.

[9] J. Jonsson and K. G. Shin, "A parametrized branch-and-bound strategy for scheduling precedence-constrained tasks on a multiprocessor system," Parallel Processing, International Conference on, vol. 0, p. 158, 1997.

[10] J. Brenner, J. van der Veen, S. Fekete, J. Oliveira Filho, and W. Rosenstiel, "Optimal simultaneous scheduling, binding and routing for processor-like reconfigurable architectures," in International Conference on Field Programmable Logic and Applications, 2006. FPL '06., 2006.

[11] M. Sadiq and S. Khan, "Optimal mapping of DSP algorithms on commercially available off-the-shelf (COTS) VLIW DSPs," Consumer Electronics, IEEE Transactions on, vol. 53, pp. 1061 -1067, 2007.

[12] K. Kuchcinski, "An approach to high-level synthesis using constraint logic programming," in Proc. 24th Euromicro Conference, Workshop on Digital System Design, Västerås, Sweden, Aug. 25-27, 1998, pp. 74-82.

[13] C. Wolinski, K. Kuchcinski, E. Raffin, and F. Charot, "Architecturedriven synthesis of reconfigurable cells," in Proc. of the 12th Euromicro conference on Digital System Design (DSD), Patras, Greece, Aug. 27-9, 2009, pp. 531-538.

[14] C. Wolinski, K. Kuchcinski, K. Martin, E. Raffin, and F. Charot, "How constrains programming can help you in the generation of optimized application specific reconfigurable processor extensions," in Proc. of The Intl. Conference on Engineering of Reconfigurable Systems and Algorithms, Las Vegas, USA, (Invited paper), Jul. 13-16, 2009.

[15] C. Wolinski, K. Kuchcinski, and E. Raffin, "Automatic design of application-specific reconfigurable processor extensions with UPaK synthesis kernel," ACM Trans. Des. Autom. Electron. Syst., vol. 15, no. 1, pp. 1-36, 2009.

[16] C. Ekelin and J. Jonsson, "Solving embedded system scheduling problems using constraint programming," Dept. of Computer Engineering, Chalmers University of Technology, Tech. Rep., 2000.

[17] L. Jówiak, N. Nedjah, and M. Figueroa, "Modern development methods and tools for embedded reconfigurable systems: A survey," Integr. VLSI J., vol. 43, pp. 1-33, 2010.

[18] J. Teich, T. Blickle, and L. Thiele, "An evolutionary approach to systemlevel synthesis," in CODES '97: Proceedings of the 5th International Workshop on Hardware/Software Co-Design. Washington, DC, USA: IEEE Computer Society, 1997, p. 167.

[19] Y. Qu, J.-P. Soininen, and J. Nurmi, "Static scheduling techniques for dependent tasks on dynamically reconfigurable devices," Journal of Systems Architecture, vol. 53, pp. 861 - 876, 2007.

[20] K. Kuchcinski, "Constraints-driven scheduling and resource assignment," ACM Transactions on Design Automation of Electronic Systems (TODAES), vol. 8, no. 3, pp. 355-383, Jul. 2003.

[21] K. Kuchcinski and R. Szymanek, "JaCoP Library. User's Guide," http: //www.jacop.eu, 2009.

[22] F. Rossi, P. v. Beek, and T. Walsh, Handbook of Constraint Programming (Foundations of Artificial Intelligence). Elsevier Science Inc., 2006. 